\title{
Estrategias para el desarrollo sostenible y sustentable del Geoparque Imbabura, Ecuador.
}

\author{
Strategies for the sustainable and sustainable development of the Imbabura \\ Geopark, Ecuador.
}

PhD. Adelfa María La Serna Gómez., ${ }^{1}$ MSc. Bertha Soraya Rhea González., ${ }^{2}$ \& MSc. Yoarnelys Vasallo Villalonga. ${ }^{3}$

\section{Resumen.}

El objetivo de la investigación es realizar una propuesta de estrategias para el desarrollo sostenible y sustentable del Geoparque Imbabura, ubicado en la provincia del mismo nombre en la sierra norte ecuatoriana, y actualmente en fase de solicitud a la UNESCO. Los métodos utilizados fueron el histórico-lógico, inductivo-deductivo y el analítico sintético para la revisión de documentos que establecen las estrategias vigentes en la zona. Asimismo, como elemento empírico, se consultó el criterio de expertos en estrategias para el desarrollo local, y se realizó el estudio comparativo de metodologías empleadas en investigaciones precedentes. Los resultados parciales de la investigación responden a los objetivos propuestos. Se comprobó el potencial que representa el desarrollo turístico para la puesta en valor del patrimonio natural, la necesidad de conciliar las estrategias existentes y de enfocar nuevas para la mayor incorporación de los sectores sociales implicados en los cambios económicos, la sostenibilidad y sustentabilidad del Geoparque.

Palabras claves: Geoparque, desarrollo, estrategia, patrimonio geológico.

\section{Abstract.}

The objective of the research is to make a proposal of strategies for the sustainable and sustainable development of the Imbabura Geopark, located in the province of the same name in the northern Ecuadorian highlands, and currently in the application phase to UNESCO. The methods used were historical-logical, inductive-deductive and analytical-

\footnotetext{
${ }^{1}$ Universidad Técnica del Norte, Facultad de Ciencias Administrativas y Económicas. Ibarra, Imbabura, Ecuador. amlaserna@utn.edu.ec

${ }^{2}$ Universidad Técnica del Norte, Facultad de Ciencias Administrativas y Económicas. Ibarra, Imbabura, Ecuador.srehag@utn.edu.ec

${ }^{3}$ Universidad Técnica del Norte, Facultad de Ciencias Administrativas y Económicas. Ibarra, Imbabura, Ecuador. yvasallo@utn.edu.ec
} 
synthetic for the review of documents that establish the current strategies in the area. Likewise, as an empirical element, the criterion of experts in strategies for local development was consulted, and a comparative study of methodologies used in previous research was carried out. The partial results of the investigation respond to the proposed objectives. The potential of tourism development for the enhancement of the natural heritage, the need to reconcile existing strategies and to focus new ones for the greater incorporation of the social sectors involved in the economic changes, sustainability and sustainability of the Geopark was proven.

Keywords: Geopark, development, strategy, geological heritage.

\section{Introducción}

La creación de los Geoparques, iniciada a mediados del decenio de 1990, constituye una opción para propiciar el desarrollo sostenible y sustentable de diferentes territorios en el mundo, pues contar con patrimonio natural de interés global no es suficiente para alcanzar el desarrollo de un área geográfica. Actualmente están aprobados 140 Geoparques, de ellos 4 transnacionales UNESCO (2018) [1].

Los Geoparques Mundiales de la UNESCO (GMU), son la designación UNESCO más reciente, establecida en 2015 como "territorios de ciencia, educación y cultura que promueven y conservan el patrimonio geológico en conexión con los demás aspectos naturales y culturales del área y se manejan bajo un enfoque comunitario y holístico de desarrollo sostenible" según UNESCO (2016) [2].

Las estrategias que se prevén para el Geoparque tienen como ámbito toda una provincia y deben considerar no solo aspectos de conservación y económicos. El proceso de desarrollo no puede entenderse plenamente si se consideran solo variables económicas, sin atender aspectos de carácter sociológico, cultural y político (Guzmán \& Romero 2008) [3].

En el proceso de crecimiento económico y social, como es el caso de los geoparques, la incorporación de la población local es decisiva para cumplir las estrategias, objetivos y acciones en cualquier proceso de crecimiento económico y social. Una referencia válida al respecto es realizada por Kliksberg (2006, p.12), cuando plantea que el grado de confianza existente entre los actores sociales, sus normas de comportamiento cívico y el nivel de asociatividad que los caracteriza; son evidenciadores de la riqueza y fortaleza del tejido social interno de una sociedad y por tanto serán elementos fundamentales para lograr un desarrollo sustentable [4].

El turismo, en diferentes modalidades, resulta esencial para la puesta en valor del patrimonio tangible e intangible en los GMU, pues genera ingresos, crea nuevas fuentes de empleo, propicia la incorporación y capacitación de sectores de menos recursos y el rescate de culturas, pero su crecimiento debe orientarse en función de las estrategias que garanticen el desarrollo sostenible.

La definición de estrategias para el desarrollo de un territorio, considerando la conservación, es realizada por entidades internacionales, a niveles local, regional, provincial o nacional, con la implementación de acciones desde los intereses y temáticas de los diferentes actores. 
La confluencia de estrategias también depende del territorio que comprende el GMU. En ocasiones estos abarcan espacios en que pueden solaparse con otro sitio designado por la UNESCO, como una Reserva de Biosfera o un sitio del Patrimonio Mundial, o pueden extenderse por territorio de más de un país.

El presente trabajo muestra resultados parciales del proyecto de investigación "Estrategias para el desarrollo turístico, sustentable e inclusivo del geoparque Imbabura" que se realiza en la Universidad Técnica del Norte (UTN) de la provincia de Imbabura, Ecuador. El objetivo del proyecto es proponer las estrategias más adecuadas para el desarrollo sostenible y sustentable del Geoparque Imbabura (GI).

La definición de dichas estrategias es compleja por la cantidad de regulaciones y factores que inciden en el marco de la provincia y más aún para su implementación y control, por la cantidad de actores que intervienen en el territorio y la diversidad de su población.

Imbabura, está situada en la Sierra Norte de Ecuador y se gestiona su clasificación como GMU, pues cuenta con amplio patrimonio geológico. En su territorio se encuentran los complejos volcánicos: Imbabura, Mojanda, Cotacachi-Cuicocha y Chachimbiro; un importante conjunto de lagunas y lagos y parte de la Reserva Ecológica Cotacachi Cayapas. Además, está considerada la provincia de mayor diversidad étnica de Ecuador.

\section{Métodos.}

La definición de una propuesta de estrategias para el desarrollo de Imbabura, se asumió con un enfoque multidimensional, se utilizaron métodos teóricos de análisis y síntesis, inductivo-deductivo, histórico-lógico, y como elemento empírico, se consultó el criterio de expertos, especialistas en estrategias para el desarrollo local y las políticas públicas, que radican en Imbabura.

Se inició la investigación con el diagnóstico, acorde al concepto de Desarrollo Sostenible propuesto por Taddei, (2011), en los ámbitos ecológico, económico, social e institucional [5]. Se analizaron indicadores económicos, las actividades con potencialidad para favorecer el desarrollo y su localización, así como las condiciones económicas, políticas y sociales de la provincia y las principales regulaciones vigentes, referidas especialmente a la conservación del patrimonio.

Se realizó una revisión bibliográfica empleando el software Atlas.ti y se utilizó el método de análisis y síntesis para documentos en los que se definen aspectos relativos a la elaboración de estrategias para el desarrollo sostenible.

Fueron consultados casos de aplicación de metodologías para definir estrategias de desarrollo local, de España y México, como elementos de comparación, entre las que aparecen la Estrategia de la Geodiversidad de la Comunidad Autónoma del País Vasco, donde se ubica un GMU, presentada por Arana y G. Monje, M. (2013), las Estrategias de desarrollo local sustentable en un área natural protegida de Baja California Sur, elaborada por Olmos y González (2011) así como la adoptada por la directiva del proyecto de Geoparque Imbabura de Silva (2003) esto permitió identificar dimensiones comunes en investigaciones precedentes.[6;7;8]. 
Se prevé determinar una línea base que permita identificar el punto de partida al momento de iniciar la aplicación de las estrategias y fijar hacia dónde se prevé llegar, no obstante, es insuficiente la información, especialmente en la actividad turística.

A partir de la información del diagnóstico y la revisión bibliográfica se elabora actualmente una matriz de potencialidades, limitaciones y problemas.

En la investigación, se parte del supuesto que Imbabura será aceptada como PMU.

\section{Resultados}

El patrimonio natural de Imbabura es significativo, tanto en sus elementos bióticos que integran la biodiversidad, como en los abióticos que componen la geodiversidad. Es una responsabilidad de sus habitantes en general y de sus autoridades garantizar su preservación. La condición de Geoparque a la que aspira puede favorecer la incorporación de más sectores sociales a participar en el desarrollo sostenible y sustentable de la provincia.

Confluyen en la provincia de Imbabura las expectativas de aplicación de normativas relacionadas con el desarrollo sostenible, tanto externas como nacionales y específicas de su reserva ecológica Cotacachi-Cayapas:

- Como parte de las normativas internacionales, se hace referencia a los Objetivos de Desarrollo Sostenible con horizonte al año 2030 [9].

- Al optar por la condición de Geoparque, se reconocen como prerrequisitos los planteamientos de requisitos de programas de la UNESCO relacionados con los GMU. [10]

- A nivel nacional, se acata lo establecido en la Constitución de Ecuador en cuanto a los derechos de la Pacha Mama (CapVII; Arts. 71-74) [11]

- Se adoptan los lineamientos del Plan Nacional de Desarrollo 2017-2021-Toda una Vida [12], en sus objetivos:

○ 3.- Mejorar la calidad de vida de la población.

- 4.- Fortalecer las capacidades y potencialidades de la ciudadanía.

○ 5.- Construir espacios de encuentro común y fortalecer la identidad nacional y las identidades diversas, la plurinacionalidad y a interculturalidad.

○ 7.- Garantizar los derechos de la Naturaleza y promover la sostenibilidad ambiental, territorial y global

- Se reconoce el Plan de Manejo de la reserva natural Cotacachi Cayapas[13].

- Se observa el Plan Estratégico de Desarrollo de Turismo Sostenible de Ecuador al 2020 [14].

- Algunos organismos como el Ministerio de Agricultura y Ganadería promueven estrategias, como la implantación de cultivos que en ocasiones atentan contra las áreas protegidas.

Los ejemplos referidos indican la prioridad de la preservación, pero se evidencia falta de coordinación sobre implementación de acciones orientadas al cumplimiento de regulaciones y estrategias vigentes en el territorio. 
En la bibliografía se identificaron actividades que pueden ser incluidas en el marco de las estrategias específicas para favorecer económicamente el incremento de emprendimientos o pequeñas inversiones, que faciliten incorporar a personas de bajos recursos, tales como: los microcréditos que se analizan en Mballa, L. (2017) [15], el desarrollo de modalidades como el turismo agroalimentario, referido por Thome (2016) [16], y la mayor introducción de las TICs, que se refiere por Micheli y Oliver (2017) respecto a las empresas de software y su vínculo con el desarrollo local [16].

La bibliografía sobre experiencias en Geoparques (G.) es limitada, pero se revisaron artículos tales como el de Espinosa (2017) sobre el G. del Distrito Minero TlalpujahuaEl Oro, en México [17]; el de Carvalho (2017) sobre el de Naturgebo de Portugal[18] y el de Rosado y Ramírez (2017) del G. de Milpa Alta en Oaxaca México [19]. En estos materiales se evidencia la importancia del turismo en diferentes modalidades referidas como el agroturismo y geoturismo y la necesidad de involucrar a la población local.

La actividad turística en Imbabura muestra potencial para la puesta en valor del patrimonio tangible e intangible, de activos y capacidades productivas de la provincia, además, puede facilitar la incorporación a nuevos empleos de sectores menos favorecidos, sin embargo, su desarrollo es incipiente. Investigaciones precedentes ratifican la preferencia en la provincia de brindar servicios al turismo de forma privada, individual y no vinculados al Sistema de Turismo del país, ni a los canales de distribución convencionales.

En la UTN, como parte de los trabajos de vinculación con la sociedad se han revisado propuestas de emprendimientos y micro, pequeñas y medianas empresas (MIPYMES) para brindar servicios turísticos, pero ha sido evidente la limitación de recursos para ejecutarlos.

Existe un grupo de trabajo del Geoparque Imbabura que se conforma en un Comité de Gestión integrado por delegados de: la Prefectura, la Alcaldía, del Ministerio de Turismo, de la Zona 1 y de la Academia. De esta forma es posible fortalecer la integración de los actores del territorio en el propósito de funcionar como GMU. La participación de actores locales se garantiza con la inscripción voluntaria de los llamados "amigos del proyecto Geoparque Imbabura" y que puede incluir a todas las personas naturales o jurídicas, públicas o privadas, organizaciones de la sociedad civil, representantes de gobiernos locales o nacional, de clubes deportivos y de todos los que estén interesados.

Las metodologías revisadas, en las que se determinan estrategias, se desarrollan en contextos muy diferentes al de Imbabura, no obstante, se identifican experiencias que pudieran extrapolarse a nuestra investigación. El grupo de trabajo del GI ya adoptó una metodología elaborada por el Instituto Latinoamericano y del Caribe de Planificación Económica y social (ILPES 2000).

\section{Discusión.}

El objetivo del proyecto de definir una propuesta de estrategias para el desarrollo sostenible y sustentable del GI. lleva implícito que esa propuesta, además de considerar las principales normativas y estrategias ya vigentes en la provincia, su conciliación y se incorporen aquellas que pueden complementar los requerimientos de un mejor desarrollo. Es por ello la importancia del diagnóstico inicial. 
Los ejemplos de las regulaciones que están vigentes con sus estrategias en la provincia indican la prioridad de la preservación, pero se evidencia falta de coordinación sobre implementación de acciones en el territorio.

Es favorable para el trabajo de definición e implementación de las estrategias del Geoparque que ya cuente con un Comité de Gestión donde participan representantes de los diferentes órganos de dirección de la provincia, esto favorecerá la definición objetiva e implementación de las estrategias.

Una mayor participación de representantes de las comunidades o grupos étnicos de Imbabura en el Comité de Gestión podrá favorecer la mayor incorporación de la sociedad en la consecución de mejores resultados económicos y sociales. Esto se propicia hoy con la incorporación de los llamados "Amigos del GI", para los que se organizan actividades en que se socializan aspectos de conservación, de los avances en las fases de aprobación del G. por la UNESCO y otros.

La actividad turística requiere especial atención en la definición de estrategias atendiendo a las características de su incipiente desarrollo, a la necesidad de implementar regulaciones para la prestación de servicios y de lograr establecer canales de distribución más apropiados.

La creación de (MIPYMES) así como de emprendimientos en la actividad turística, por parte de los sectores menos favorecidos se ve limitada por insuficientes recursos y desconocimiento de alternativas de financiamiento.

\section{Conclusiones}

$>$ La definición de las estrategias para el G.I. debe considerar la conciliación de las regulaciones y estrategias que hoy están vigentes en la provincia, precisando las que puedan estar faltando, especialmente relacionadas con la mayor incorporación de los sectores que pueden protagonizar mayores cambios económicos y sociales.

$>$ La asertividad de las estrategias que se definan para el G.I. también estará en dependencia de la posibilidad de que la entidad de gestión de este, cuente con el apoyo de las autoridades nacionales y locales para lograr su implementación, lo cual ya se observa en la composición del Comité de Gestión.

$>$ El impacto de implementar las estrategias definidas en el Geoparque será mayor en la medida en que se incorporen más sectores sociales implicados en los posibles cambios económicos y sociales.

$>$ La actividad turística debe priorizarse en las estrategias, porque propicia el crecimiento económico y social y puede incorporar modalidades que faciliten en breve plazo la puesta en valor de los atractivos, para ello es conveniente valorar objetivamente las condiciones a crear, como la de ofrecer los microcréditos, imprescindibles para desarrollar emprendimientos o MYPIMES por parte de los sectores menos favorecidos. 


\section{Referencias Bibliográficas}

[1] UNESCO. Lista de Geoparques Globales de la UNESCO. París.2018 [Consulta 23-82018] Disponible en: http://www.unesco.org/new/en/naturalsciences/environment/earth-sciences/unesco-global-geoparks/list-of-unescoglobal-geoparks/

[2] UNESCO Statutes of the international geoscience and geoparks programme. París: 2016 [Consulta 23-8-2018] Disponible en: http://unesdoc.unesco.org/images/0026/002606/260675s.pdf

[3] GUZMÁN, J.; ROMERO, Crecimiento económico, gobernabilidad democrática y desarrollo social: un enfoque integrador. Problemas del Desarrollo. Revista Latinoamericana de Economía [en línea] 2008, 39 (Abril-Junio): [Consulta: 26 de agosto de 2018] Disponible en:<http://www.redalyc.org/articulo.oa?id=11820161005> ISSN 0301-7036

[4] KLIKSBERG, Bernardo, CAPITAL SOCIAL Y CULTURA, CLAVES DEL DESARROLLO. Cuadernos Latinoamericanos de Administración [en linea] 2006, II (Enero-Junio) : [Consulta: 26 de agosto de 2018] Disponible en:<http://agricola-www.redalyc.org/articulo.oa?id=409634344002> ISSN $1900-5016$

[5] TADDEI DIEZ, Lopoldo José. Posibilidades y retos del desarrollo sostenible en la región Tacna. [en línea] 9 (38-51): 2011. [Consulta: 23 de septiembre de 2018]. Disponible en: http://www.eumed.net/tesis-doctorales/2011/ljtd/index.htm. ISBN-13: 978-84-694-8771-6

[6] ARANA, G. MONJE, M. La estrategia de geodiversidad de la comunidad autónoma del País Vasco.Cuadernos del Museo Geominero, $\mathrm{n}^{\circ} 15$ Instituto Geológico Minero de España: 1-7, 2013 ISBN XXX-XX-XXXX-XXX-X.

[7] OLMOS-MARTINEZ, E y GONZALEZ-AVILA, ME. Estrategias de desarrollo local sustentable en un área natural protegida de Baja California Sur. Universidad y ciencia [online]. 2011, vol.27, n.3 [Consulta: 2018-08-26], pp.281-298. $29792011000300004 \& \operatorname{lng}=e s \& n r m=i s o>$. ISSN 0186-2979.

[8] SILVA, I. Metodología para la elaboración de estrategias de desarrollo local.[en línea] Instituto Latinoamericano y del Caribe de Planificación Económica y Social (ILPES) CEPAL. Santiago de Chile, noviembre de 2003 [Consultado 238-2018] Disponible en: https://www.cepal.org/publicaciones/xml/7/13867/sgp42.pdf 
[9] ONU Agenda 2030 y los Objetivos de Desarrollo Sostenible Una oportunidad para América Latina y el Caribe. 2016 (Consulta 23-9-2018) Disponible en: http://www.sela.org/media/2262361/agenda-2030-y-los-objetivos-dedesarrollo-sostenible.pdf

[10] UNESCO Directrices y criterios para Parques Nacionales interesados en recibir asistencia de la UNESCO para integrar la Red Mundial de Geoparques (GGN) [en línea] París 2010.

[11] Autor corporativo Asamblea Nacional. Constitución de la República de Ecuador. 2008.

[12] Autor corporativo. Secretaría Nacional de Planificación y Desarrollo, SENPLADES. El Plan Nacional de Desarrollo "Plan Nacional de Desarrollo 2017-2021.Toda una Vida" Ecuador 2017

[13] Autor corporativo. Ministerio del Ambiente del Ecuador. Plan de Manejo de la Reserva Ecológica Cotacachi Cayapas. Proyecto GEF Ecuador: Sistema Nacional de Áreas Protegidas (SNAP-GEF). Quito. 2007

[14] Autor corporativo. Equipo PLANDETUR 2020 del Ministerio de Turismo de Ecuador et al.Plan Estratégico de Desarrollo de Turismo Sostenible de Ecuador al 2020. Ecuador 2007. [Consulta: 22 septiembre de 2018]. Disponible en: http://www.turismo.gob.ec/wp-content/uploads/downloads/ 2013/02/PLANDETUR - 2020.pdf

[15] MBALLA, Louis Valentin. Desarrollo local y microfinanzas como estrategias de atención a las necesidades sociales: un acercamiento teórico conceptual. Rev. Mexicana de ciencias políticas [online]. 2017, vol.62, n.229 [citado 2018-0826], pp.101-127. Disponible en: <http://www.scielo.org.mx/scielo.php?script=sci_arttext\&pid=S0185$19182017000100101 \& \operatorname{lng}=$ es\&nrm=iso>. ISSN 0185-1918.

[16] THOME-ORTIZ, Humberto. Turismo agroalimentario y nuevos metabolismos sociales de productos locales. Rev. Mex. Cienc. Agríc [online]. 2015, vol.6, n.6 [citado 2018-09-26], pp.1373-1386. Disponible en: <http://www.scielo.org.mx/scielo.php?script=sci_arttext\&pid=S200709342015000600018\&lng=es\&nrm=iso >. ISSN 2007-0934

[16] 16]MICHELI, Jordy y OLIVER, Rubén. Empresas de software en México y sus vínculos de desarrollo local. Prob. Des [online]. 2017, vol.48, n.190 [Consulta 2018-09-26], pp.37-59.

Disponible en: <http://www.scielo.org.mx/scielo.php?script=sci_arttext\&pid=S030170362017000300037\&lng=es\&nrm=iso>. ISSN 0301-7036. 
[17] ESPINOSA-RODRIGUEZ, Luis Miguel. Geoparque en el Distrito Minero Tlalpujahua-El Oro. CienciaUAT [online]. 2017, vol.11, n.2 [Consulta 2018-0922], pp.24-45.

Disponible

en:

<http://www.scielo.org.mx/scielo.php?script=sci_arttext\&pid=S200778582017000100024\&lng=es\&nrm=iso>. ISSN 2007-7858.

[18] NETO de CARVALHO,C., Tourism in the Naturtejo Geopark, Under the Auspices of UNESCO, as Sustainable Alternative to the Mining of Uranium at Nisa (Portugal). Procedia Earth and Planetary Science 8 [on line]. 2014, [Consulta 2018-8-20],p.p. $86 \quad-\quad 92 . \quad$ Disponible en: https://www.sciencedirect.com/science/article/pii/S1878522014000198

[19] ROSADO-GONZALEZ, Emmaline Montserrat y RAMIREZ-MIGUEL, Xóchitl.Importancia del trabajo comunitario participativo para el establecimiento del Geoparque Mundial de la UNESCO Mixteca Alta, Oaxaca, México. Invest. Geog [online]. 2017, n.92 [citado 2018-09-27]. Disponible en: $<$ http://www.scielo.org.mx/scielo.php?script=sci_arttext\&pid=S0188-

$46112017000100012 \& \operatorname{lng}=\mathrm{es} \& \mathrm{nrm}=$ iso $>$.

ISSN

2448-7279. http://dx.doi.org/10.14350/rig.59435. 


\section{Para citar el artículo indexado.}

La Serna A., Rhea B. \& Vasallo Y. (2017). Estrategias para el desarrollo sostenible y sustentable del Geoparque Imbabura, Ecuador. Revista electrónica Explorador Digital 1(1), 61-70. Recuperado desde:

http://cienciadigital.org/revistacienciadigital2/index.php/exploradordigital/article/view/317/7 $\underline{29}$

\section{Ciencia \\ Digital \\ Editorial}

El artículo que se publica es de exclusiva responsabilidad de los autores y no necesariamente reflejan el pensamiento de la Revista Explorador Digital.

El articulo queda en propiedad de la revista y, por tanto, su publicación parcial y/o total en otro medio tiene que ser autorizado por el director o editor de la Revista Explorador Digital.
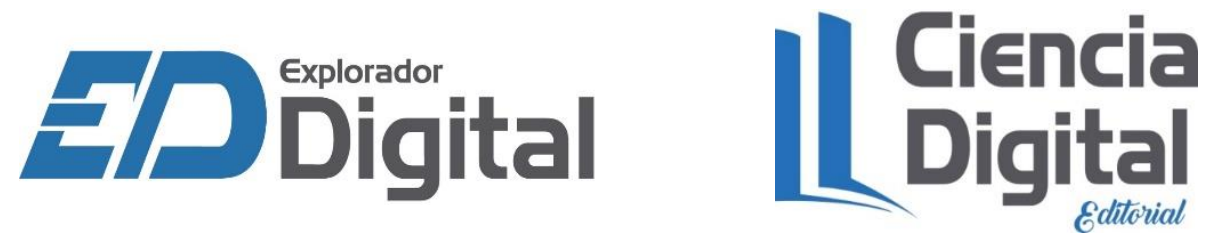